\title{
ON THE GLOBAL SOLVABILITY OF A CLASS OF FOURTH-ORDER NONLINEAR BOUNDARY VALUE PROBLEMS
}

\section{M.B.M. ELGINDI}

Department of Mathematics

University of Wisconsin - Eau Claire

Eau Claire, Wisconsin 54702 U.S.A.

elgindmb@uwec edu

\section{ZHENGYUAN GUAN}

Department of Mathematics

University of Wisconsin - Eau Claire

Eau Claire, Wisconsin 54702 U.S.A

guanz@uwec.edu

(Received May 16, 1995)

\begin{abstract}
This paper is concerned with the global solvability of a class of fourth-order nonlinear boundary value problems that govern the deformation of an elastic beam which is acted upon by axial compression, lateral forces and is in contact with a semi-infinite medium acting as a foundation For certain ranges of the acting axial compression force, the solvability of the equations follows from the coercivity of their linear parts. Beyond these ranges this coercivity is lost It is shown here that the coercivity which ensures the global solvability can be generated by the nonlinear parts of the equations for a certain type of foundation.
\end{abstract}

KEY WORDS AND PHRASES: Global solvability, fourth-order nonlinear boundary value problems, homogeneous nonlinearity, Leray-Schauder fixed point theorem, coercivity 1991 AMS SUBJECT CLASSIFICATION CODES: 49G99, 73H05, 73K15.

\section{INTRODUCTION}

In this paper we are concerned with the global solvability of the fourth-order nonlinear boundary value problems which govern the equilibrium states of a beam-column. The source of the nonlinearity comes from a nonlinear lateral constraint (foundation). The equilibrium equation is formulated as a fourth-order nonlinear differential equation. Different boundary conditions, corresponding to various ways in which the ends of the beam may be supported, will be considered. The proof of the existence of solution is based upon a corollary of Leray-Schauder Fixed Point Theorem, which we will state in Section 2 of this paper, together with an idea which originated in [2].

Existence of solutions of the boundary value problems considered in this paper has been the subject of several recent papers. The reader is referred to [1], [3] and [4] and the references therein for an extensive account on the subject. In all these works the necessary coercivity condition, which ensures the existence of solutions, was derived from the linear parts of the equations. Since this coercivity is lost beyond certain critical value of the compressive force, these papers failed to obtain any existence theorem of global nature. In this paper, on the other hand, the coercivity is generated by the nonlinear part of the equations and the existence results we obtain are global in nature.

Following [1] the differential equation which governs the lateral displacement $y(x)$ is

$$
y^{\prime \prime \prime \prime}+\lambda y^{\prime \prime}+k y+G\left(x, y, y^{\prime}, y^{\prime \prime}\right)=f(x),
$$

where $\lambda$ and $k$ are real parameters representing the axial compression and the modulus of the foundation, respectively, $k y+G\left(x, y, y^{\prime}, y^{\prime \prime}\right)$ represents the nonlinear foundation, and $f(x)$ represents the acting lateral force. We consider (1.1) along with one of the following six sets of boundary conditions. 


$$
\begin{aligned}
& y(0)=y^{\prime \prime}(0)=y(1)=y^{\prime \prime}(1)=0 \\
& y(0)=y^{\prime \prime}(0)=y(1)=y^{\prime}(1)=0 \\
& y(0)=y^{\prime \prime}(0)=y^{\prime}(1)=y^{\prime \prime \prime}(1)=0 \\
& y(0)=y^{\prime}(0)=y(1)=y^{\prime}(1)=0 \\
& y(0)=y^{\prime}(0)=y^{\prime \prime}(1)=y^{\prime \prime \prime}(1)=0 \\
& y(0)=y^{\prime}(0)=y^{\prime}(1)=y^{\prime \prime \prime}(1)=0
\end{aligned}
$$

which represent the following cases: both ends are simply-supported, one end is simply-supported and the other is fixed; one end is simply-supported and the other is sliding clamped; both ends are fixed; one end is fixed and the other is free and one end is fixed and the other is sliding clamped.

The rest of this paper is organized in three sections. In Section 2 we state the conditions and the Lemmas, on which the proof of the main result of this paper will be based, and we obtain some preliminary results In Section 3 we state and prove the main result of the paper. In Section 4 some results concerning the uniqueness of the solutions are obtained.

\section{ASSUMPTIONS AND PRELIMINARY RESULTS}

Throughout the rest of this paper we will use the following notations

$$
\begin{gathered}
W^{k}=\left\{y:[0,1] \rightarrow R: y^{(j)} \in A C[0,1], j=0,1, \ldots, k-1 \text { and } y^{(k)} \in L^{2}(0,1)\right\}, \\
\|y\|_{k}^{2}=\sum_{j=0}^{k}\left\|y^{(\jmath)}\right\|_{L_{2}}^{2}, y \in W^{k}, \\
D\left(L_{\imath}\right)=\left\{y \in W^{4}: y \text { satisfies the ith boundary conditions }(1 . i), i=2,3, \ldots, 7\right\}, \\
L_{\imath}: D\left(L_{2}\right) \rightarrow L^{2}(0,1) \text { is defined by } L_{\imath}(y) \equiv y^{\prime \prime \prime \prime} .
\end{gathered}
$$

We make the following assumptions.

$\mathrm{H}(1) . \quad f \in L^{2}(0,1)$;

$\mathrm{H}(2) . \quad G\left(x, y, y^{\prime}, y^{\prime \prime}\right)=g(y)+h\left(x, y, y^{\prime}, y^{\prime \prime}\right)$, where both $g$ and $h$ are continuous, and the map $H:[0,1] \times W^{2} \rightarrow L^{2}(0,1)$ defined by $H(x, y)=h\left(x, y, y^{\prime}, y^{\prime \prime}\right)$ is continuous Furthermore, we assume:

a. there exists $p>1$ such that $g(r x)=r^{p} g(x)$, for $r, x \in R$ with $r>0$;

b. for any $y \in W^{2}, \int_{0}^{1} g(y) y d x \geq 0$; and $\int_{0}^{1} g(y) y d x=0$ iff $y=0$;

c. $\int_{0}^{1} h\left(x, y, y^{\prime}, y^{\prime \prime}\right) y d x \geq 0, y \in W^{2}$.

The proof of our main result of the next section consists of verifying the conditions of a corollary of Leray-Schauder Fixed Point Theorem which we state here as the following lemma.

LEMMA 2.1. Let $B$ be a Banach space and $K: B \rightarrow B$ be a compact operator. Suppose that there exists a priori bound $m>0$ such that every solution of $y-t K y=0$, for $t \in[0,1]$, satisfies $\|y\| \leq m$. Then $K$ has a fixed point $y$ with $\|y\| \leq m$.

We collect some preliminary results which we will use in Section 3 in the following lemma.

LEMMA 2.2. For each $L, j=2,3, \ldots, 7$, the following are true:

A. $L_{\jmath}$, as an operator on $L^{2}(0,1)$, is densely defined and self-adjoint;

B. $\|y\|_{L_{2}} \leq C_{\jmath}^{-1}\left\|y^{\prime \prime}\right\|_{L_{2}}$, for $y \in D\left(L_{\jmath}\right)$, with $C_{2}=\pi^{2} ; C_{3}=\frac{\pi^{2}}{2} ; C_{4}=\frac{\pi^{2}}{4} ; C_{5}=\pi^{2} ; C_{6}=\frac{\pi^{2}}{4}$; and $C_{7}=\frac{\pi^{2}}{2}$;

C. for any $y \in D\left(L_{\jmath}\right), L_{\jmath} y=0$ iff $y=0$;

$D$. there exist unique $\psi_{\jmath}: L^{2}(0,1) \rightarrow W^{4}$ such that $L_{\jmath}\left(\psi_{j}(h)\right)=h$ for any $h \in L^{2}(0,1)$, and $\psi_{j}: L^{2}(0,1) \rightarrow W^{4}$ is bounded;

E. $\bar{K}_{\jmath}: L^{2}(0,1) \rightarrow W^{2}$ defined by $\bar{K}_{\jmath}=i \cdot \psi_{\jmath}$, where $i: D\left(L_{\jmath}\right) \rightarrow W^{2}$ denotes the identity map, is compact.

The proofs of (A)-(E) are direct and are therefore omitted. For some of the estimates in (B), one needs to use Wirtinger's Inequalities [5]. 


\section{GLOBAL EXISTENCE OF SOLUTIONS}

In this section we consider the global solvability of the six boundary value problems consisting of the differential equation (1.1) and one of the six sets of boundary conditions (1.2)-(1 7) in the following theorem.

THEOREM 3.1. Under the assumption $H(1)$ and $H(2)$, the boundary value problem consisting of (1.1) and (1.J), $j=2,3, \ldots, 7$, has at least one solution for each $k \geq 0$ and each $\lambda \geq 0$

PROOF. The boundary value problem (1.1), (1.j), $2 \leq j \leq 7$, can be written as

$$
y=K_{\jmath} y
$$

where

$$
K_{\jmath} y=-\bar{K}_{\jmath}\left[\lambda y^{\prime \prime}+k y+G\left(x, y, y^{\prime}, y^{\prime \prime}\right)-f(x)\right],
$$

$K_{\jmath}: W^{2} \rightarrow W^{2}$ is compact, and $\bar{K}$, is as in Lemma 2.2 We prove the existence of a solution of (3 1$)$ by verifying the conditions of Lemma 2.1 .

Assume that the solutions of $y-t K, y=$ are not uniformly bounded with respect to $t \in[0,1]$ Then there exist sequence $\left\{t_{n}\right\} \subset(0,1)$ and $\left\{y_{n}\right\} \subset W^{2}$ such that

$$
y_{n}=t_{n} K, y_{n}, \quad n \geq 1
$$

and $\left\|y_{n}\right\|_{2} \rightarrow \infty$ as $n \rightarrow \infty$.

From (3.2), it follows that each $y_{n}$ satisfies

$$
y_{n}^{\prime \prime \prime \prime}+t_{n} \lambda y_{n}^{\prime \prime}+t_{n} k y_{n}+t_{n} G\left(x, y_{n}, y_{n}^{\prime}, y_{n}^{\prime \prime}\right)=t_{n} f(x)
$$

with $y_{n} \in D\left(L_{j}\right)$, which in turn implies (upon multiplying both sides of the equation by $y_{n}$, integrating by parts and using the boundary conditions)

$$
\left\|y^{\prime \prime}\right\|_{L_{2}}^{2}+t_{n} \lambda \int_{0}^{1} y_{n}^{\prime \prime} y_{n} d x+t_{n} k\left\|y_{n}\right\|_{L_{2}}^{2}+t_{n} \int_{0}^{1} G\left(x, y_{n}, y_{n}^{\prime}, y_{n}^{\prime \prime}\right) y_{n} d x=t_{n} \int_{0}^{1} f y_{n} d x
$$

Set $z_{n} \equiv \frac{y_{n}}{\left\|y_{n}\right\|_{2}}$, then $\left\{z_{n}\right\} \subset W^{2}$ is a bounded sequence, and since a bounded set of $W^{2}$ is weakly relatively compact, it follows that there exists a subsequence of $\left\{z_{n}\right\}$, which we call $\left\{z_{n}\right\}$, that converges weakly in $W^{2}$. By the fact that the imbedding $i: D\left(L_{\jmath}\right) \subset W^{2} \rightarrow C^{1}[0,1]$ is compact, it follows that there exists a subsequence of $\left\{z_{n}\right\}$, which we call $\left\{z_{n}\right\}$ again, that converges strongly in $C^{1}[0,1]$ to some $z_{0} \in C^{1}[0,1]$

From (3.3) and assumption $\mathrm{H}(2)$ we obtain

$$
\begin{aligned}
t_{n} \int_{0}^{1} g\left(y_{n}\right) y_{n} d x= & -\left\|y^{\prime \prime}\right\|_{L_{2}}^{2}-t_{n} \lambda \int_{0}^{1} y_{n}^{\prime \prime} y_{n} d x \\
& -t_{n} k\left\|y_{n}\right\|_{L_{2}}^{2}-t_{n} \int_{0}^{1} h\left(x, y_{n}, y_{n}^{\prime}, y_{n}^{\prime \prime}\right) y_{n} d x+t_{n} \int_{0}^{1} f y_{n} d x \\
& \leq t_{n} \lambda\left\|y_{n}^{\prime \prime}\right\|_{L_{2}}\left\|y_{n}\right\|_{L_{2}}+t_{n} \int_{0}^{1} f y_{n} d x \\
& \leq t_{n} C_{\jmath}^{-1} \lambda\left\|y_{n}^{\prime \prime}\right\|_{L_{2}}^{2}+t_{n}\|f\|_{L_{2}}\left\|y_{n}\right\|_{L_{2}} .
\end{aligned}
$$

Using (3.4) and homogeneity of $g$ we obtain (since $p>1$ )

$$
0 \leq \int_{0}^{1} g\left(z_{n}\right) z_{n} d x \leq \frac{\lambda\left\|y^{\prime \prime}\right\|_{L_{2}}^{2}}{C_{\jmath}\left\|y_{n}\right\|_{2}^{p+1}}+\frac{\|f\|_{L_{2}}\left\|y_{n}\right\|_{L_{2}}}{\left\|y_{n}\right\|_{2}^{p+1}} \rightarrow 0
$$

as $n \rightarrow \infty$ Since $g$ is continuous, it follows from (3.5) that

$$
\int_{0}^{1} g\left(z_{0}\right) z_{0} d x=0
$$


which, in view of assumption $\mathrm{H}(2)(\mathrm{b})$, implies that $z_{0}=0$, and $z_{n} \rightarrow 0$ in $C^{1}[0,1]$.

On the other hand, from (3.3) we have

$$
\begin{aligned}
\frac{\left\|y_{n}^{\prime \prime}\right\|_{L_{2}}^{2}=}{\left\|y_{n}\right\|_{2}^{2}}= & -t_{n} \lambda \int_{0}^{1} \frac{y_{n}^{\prime \prime} y_{n}}{\left\|y_{n}\right\|_{2}^{2}} d x-t_{n} k \frac{\left\|y_{n}\right\|_{L_{2}}^{2}}{\left\|y_{n}\right\|_{2}^{2}} \\
& -t_{n} \int_{0}^{1} G\left(x, y_{n}, y_{n}^{\prime}, y_{n}^{\prime \prime}\right) \frac{y_{n}}{\left\|y_{2}\right\|_{2}^{2}} d x+\frac{t_{n}}{\left\|y_{n}\right\|_{2}^{2}} \int_{0}^{1} f y_{n} d x \\
& \leq-t_{n} \lambda\left[z_{n}^{\prime}(1) z_{n}(1)-z_{n}^{\prime}(0) z_{n}(0)\right]+t_{n} \lambda \int_{0}^{1}{z^{\prime}}_{n}^{2} d x+t_{n} \frac{\|f\|_{L_{2}}\left\|y_{n}\right\|_{L_{2}}}{\left\|y_{n}\right\|_{2}^{2}}
\end{aligned}
$$

which implies that (by the fact $z_{n} \rightarrow 0$ in $C^{1}[0,1]$ )

$$
\frac{\left\|y_{n}^{\prime \prime}\right\|_{L_{2}}^{2}}{\left\|y_{n}\right\|_{2}^{2}} \rightarrow 0 .
$$

However, (from part (B) of Lemma 2.2 and $\left\|y_{n}^{\prime}\right\|_{L_{2}} \leq\left\|y_{n}^{\prime \prime}\right\|_{L_{2}}$ for $y \in D\left(L_{\jmath}\right)$ ) we have

$$
\left\|y_{n}\right\|_{2}^{2}=\left\|y_{n}\right\|_{L_{2}}^{2}+\left\|y_{n}^{\prime}\right\|_{L_{2}}^{2} \leq\left(2+C_{\jmath}^{-2}\right)\left\|y_{n}^{\prime \prime}\right\|_{L_{2}}^{2} \text {, }
$$

and this contradicts (3.6). This completes the proof

\section{UNIQUENESS}

Assuming that $G\left(x, y, y^{\prime}, y^{\prime \prime}\right)$ satisfies the condition

$\mathrm{H}(3) . \int_{0}^{1}\left[G\left(x, y, y^{\prime}, y^{\prime \prime}\right)-G\left(x, z, z^{\prime}, z^{\prime \prime}\right)\right](y-z) d x>0$, for all $y, z \in W^{2}$, we obtain the following result on the uniqueness of the solution.

THEOREM 4.1. Assume $H(3)$, the solution of the boundary value problem (1.1) and (1.j), $2 \leq j \leq 7$, is unique, provided that $k \leq C_{\jmath}^{2}$ and $\lambda<C_{\jmath}+\frac{k}{C}$, or $k>C_{\jmath}^{2}$ and $\lambda<2 \sqrt{k}$.

PROOF. Let $y$ and $z$ be two solutions of the boundary value problem. Set $w=y-z$ and assume that $w \neq 0 \quad w$ satisfies the equation

$$
w^{\prime \prime \prime \prime}+\lambda w^{\prime \prime}+k w+G\left(x, y, y^{\prime}, y^{\prime \prime}\right)-G\left(x, z, z^{\prime}, z^{\prime \prime}\right)=0
$$

and the boundary condition (1.j). Let $A=\left\|w^{\prime \prime}\right\|_{L_{2}}$ and $B=\|w\|_{L_{2}}$. Upon multiplying equation (4.1) by $w$ and integrating by parts, using the boundary conditions, Hölder's inequality and $\mathrm{H}(3)$, we obtain

$$
A^{2}-\lambda A B+k B^{2} \leq 0
$$

If $k \leq C_{\jmath}^{2}$ and $\lambda<C_{\jmath}+\frac{k}{c_{\jmath}}$, we have

$$
\begin{aligned}
& A^{2}-\lambda A B+k B^{2} \\
& >A^{2}-\left(C_{\jmath}+\frac{k}{C_{\jmath}}\right) A B+k B^{2} \\
& =A\left(A-C_{\jmath} B\right)-\frac{k}{C_{\jmath}} B\left(A-C_{\jmath} B\right) \\
& =\left(A-C_{\jmath} B\right)\left(A-\frac{k}{C_{\jmath}} B\right) \\
& \geq 0
\end{aligned}
$$

since $w \in D\left(L_{\jmath}\right), A \geq C, B$, and $k \leq C_{\jmath}^{2}$. This contradicts the inequality (4.2)

If $k>C_{\jmath}^{2}$ and $\lambda<2 \sqrt{k}$, we have 


$$
\begin{aligned}
& A^{2}-\lambda A B+k B^{2} \\
& =\left(A^{2}-\lambda A B+\left(\frac{\lambda}{2}\right)^{2} B^{2}\right)+\left(k-\left(\frac{\lambda}{2}\right)^{2}\right) B^{2} \\
& =\left(A-\frac{\lambda}{2} B\right)^{2}+\left(k-\left(\frac{\lambda}{2}\right)^{2}\right) B^{2} \\
& >0
\end{aligned}
$$

since $\lambda<2 \sqrt{k}$ implies $k-\left(\frac{\lambda}{2}\right)^{2}>0$ This again contradicts (4.2).

Thus $w=0$ This proves the theorem

REMARKS. 1. From the proof of Theorem 31 , we can see that the assumptions $k \geq 0$ and $\lambda \geq 0$ are not needed. It is due to the physics nature of the problem, we assume $k \geq 0$ and $\lambda \geq 0$.

2. When the foundation of beam is not uniform, $k$ could depend on $x$, say, $k=k(x)$. Assume $k(x)$ is continuous. Theorem 31 is still true. Let $k_{m}=\min \{k(x), x \in[0,1]\}$, Theorem 4.1 is also true, with $k$ replaced by $k_{m}$.

3. With a trivial modification of the proof, we can replace assumption $\mathrm{H}(2)$ (c) by the following condition

$\mathrm{H}(2) c^{\prime} \cdot \int_{0}^{1} G\left(x, y, y^{\prime}, y^{\prime \prime}\right) y d x \geq 0$ and

$$
\frac{\int_{0}^{1} h\left(x, y, y^{\prime}, y^{\prime \prime}\right) y d x}{\|y\|_{2}^{p+1}} \rightarrow 0 \text { as }\|y\|_{2} \rightarrow \infty .
$$

4. It is quite clear that most of the functions $G$ which are of interest physically satisfy our assumption $\mathrm{H}(2)(\mathrm{a})$, (b) and (c) or $\left(\mathrm{c}^{\prime}\right)$, and $\mathrm{H}(3)$. For example, $G\left(x, y, y^{\prime}, y^{\prime \prime}\right)=y^{3}$ satisfies all these assumptions. More generally, $G\left(x, y, y^{\prime}, y^{\prime \prime}\right)=c_{3} y^{3}+c_{5} y^{5}+c_{y} y^{7}+\cdots+c_{2 n+1} y^{2 n+1}$ with $G\left(x, y, y^{\prime}, y^{\prime \prime}\right) y \geq 0$ and $c_{2 n+1}>0,\left(c_{\imath}\right.$ are constants, $\left.i=3,5, \ldots, 2 n+1\right)$ satisfies our assumptions $\mathrm{H}(2)$ (a), (b) and ( $\left.\mathrm{c}^{\prime}\right)$, if we take $g(y)=c_{2 n+1} y^{2 n+1}$. If we further assume $c_{\imath} \geq 0$ for $i=3,5, \ldots, 2 n-1, G$ also satisfies $\mathrm{H}(3)$.

\section{REFERENCES}

[1] ELGINDI, M.B.M. and YEN, D.H.Y., On the existence of equilibrium states of an elastic beam on a nonlinear foundation, Internat. J. Math. \& Math. Sci. 16, 1 (1993), 193-198.

[2] GUAN, Z., Solvability of semilinear equations with compact perturbations of operators of monotone type, Proc. Amer. Math. Soc. 121, 1 (1994), 93-102.

[3] GUPTA, C.P., Existence and uniqueness theorems for the bending of an elastic beam equation, Applicable Analysis 26 (1988), 289-304.

[4] GUPTA, C.P., Existence and uniqueness results for the bending of an elastic beam equation at resonance, J. Math. Anal. Appl. 135 (1988), 208-225.

[5] HARDY, G.H., LITTLEWOOD, J.E. and POLYA, G., Inequalities, Cambridge University Press, London and New York, 1952. 


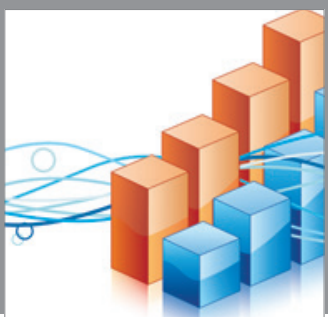

Advances in

Operations Research

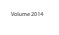

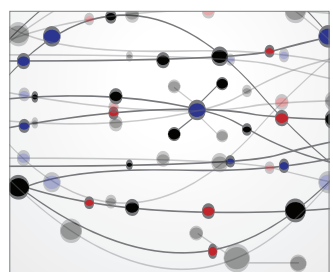

\section{The Scientific} World Journal
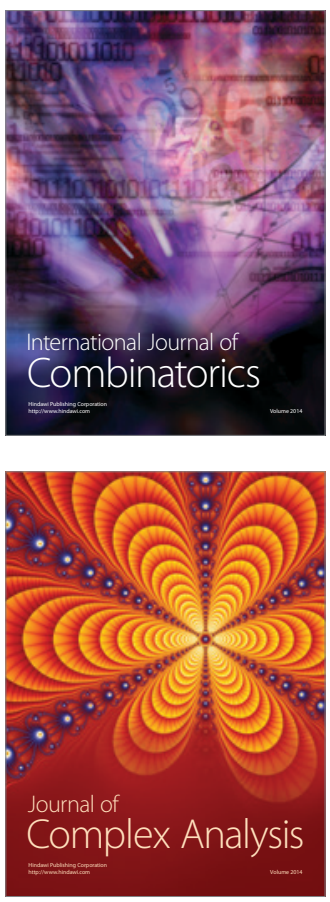

International Journal of

Mathematics and

Mathematical

Sciences
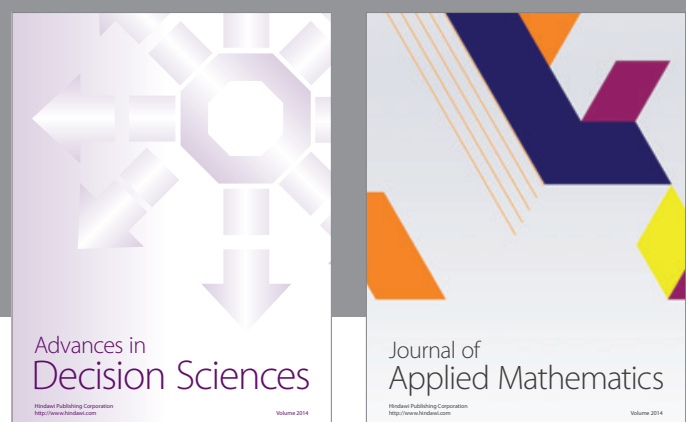

Journal of

Applied Mathematics
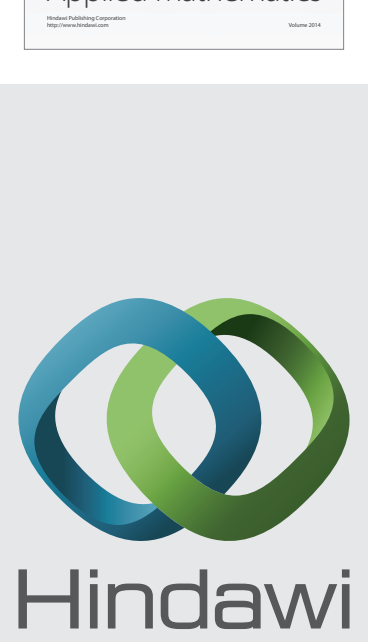

Submit your manuscripts at http://www.hindawi.com
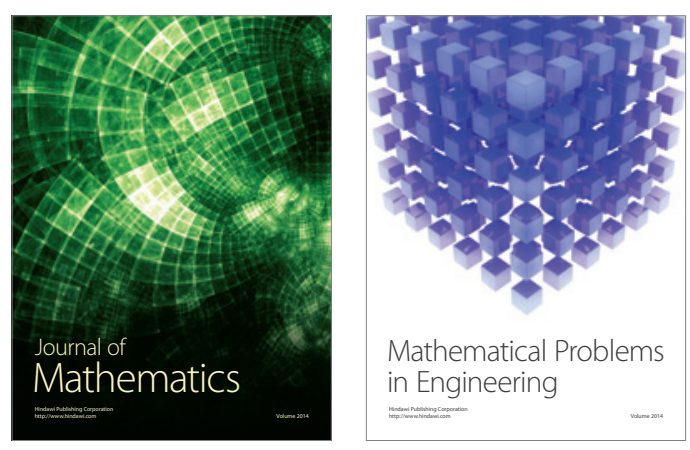

Mathematical Problems in Engineering
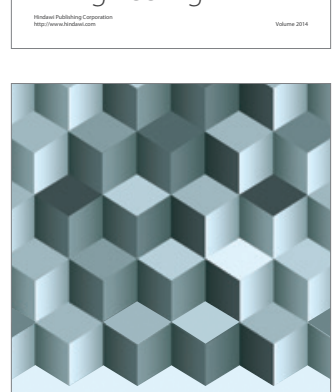

Journal of

Function Spaces
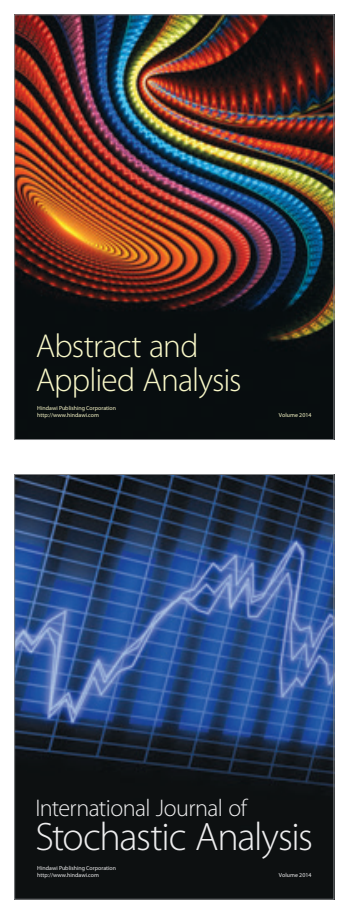

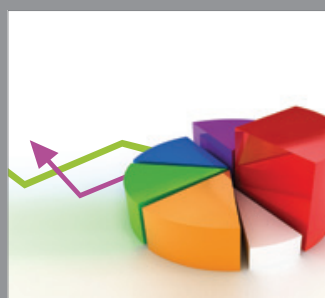

ournal of

Probability and Statistics

Promensencen
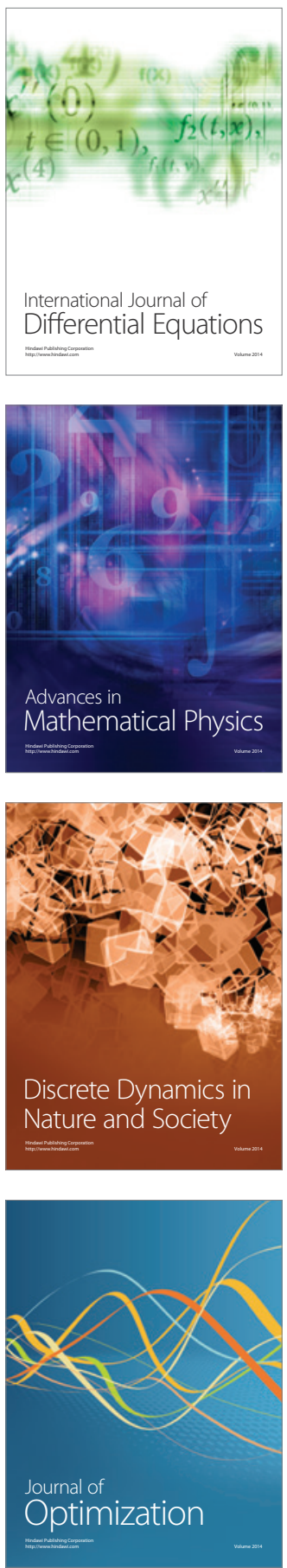